Research Paper

\title{
B cell lymphoma with different metabolic characteristics show distinct sensitivities to metabolic inhibitors
}

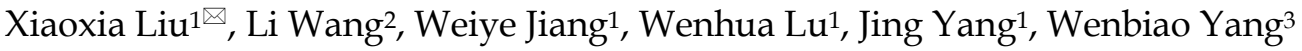 \\ 1. Sun Yat-Sen University Cancer Center, State Key Laboratory of Oncology in Southern China, Collaborative Innovation Center for Cancer Medicine, \\ Guangzhou, 510275, P.R. China. \\ 2. Department of Pharmacy, Zhujiang Hospital, Southern Medical University, Guangzhou 510282, China. \\ 3. Beijing Zhongkang of Chinese and Western medicine hospital, Beijing, 100077, P.R. China. \\ $\square$ Corresponding author: Xiaoxia Liu, PhD., Sun Yat-Sen University Cancer Center, State Key Laboratory of Oncology in Southern China, Collaborative \\ Innovation Center for Cancer Medicine, Guangzhou, 510275, P.R. China. Tel: +86(0)2087343171; E-mail: 1xx913@163.com \\ (1) Ivyspring International Publisher. This is an open access article distributed under the terms of the Creative Commons Attribution (CC BY-NC) license \\ (https:// creativecommons.org/licenses/by-nc/4.0/). See http://ivyspring.com/terms for full terms and conditions.
}

Received: 2017.12.13; Accepted: 2018.02.06; Published: 2018.04.12

\begin{abstract}
Purpose: Cancer cells exhibit profound alterations in their metabolism (abnormal glucose and glutamine metabolism). Targeting cancer metabolism is a promising therapeutic strategy. Lymphoma can be classified into many different types and it is very complicated. Therefore, in this paper, we want to know whether the B cell lymphoma cells with different metabolic characteristics have distinct sensitivities to metabolic inhibitors.

Methods: We classified $9 \mathrm{~B}$ cell lymphoma cell lines into different metabolic subtypes according to the dependency on glutamine and glucose. Then we detected the OCR, ECAR, glucose consumption and lactate production, mitochondrial content and growth rate. And we also determined the IC50 of these 9 cell lines to metabolic inhibitors.

Results: According to the dependency on glutamine and glucose, we successfully classified three distinct metabolic subtypes in B cell lymphoma cell lines, one subtype was defined glutamine and glucose equally utilized subtype $(\mathrm{GLN}=\mathrm{Glu})$, whereas the other two subtypes were $\mathrm{GLN}$-addicted and Glu-dependent. And these three subtypes showed striking differences in glucose and glutamine utilization, glycolysis and mitochondrial function, and proliferation rate. GLN-addicted and Glu-dependence subtypes also showed differences in cell sensitivity to inhibitors of glutamine and glycolysis metabolism, respectively. However, $G L N=G l u$ subtype seems minimal sensitive to glycolytic and glutaminolytic inhibitors, and with high proliferation rate.

Conclusions: The cells rely more on glucose/gltamine have a stronger sensitivity to glucose/glutamine depletion or glycolysis/ glutaminolysis inhibition and a lessened sensitivity to glutaminolysis/glycolysis inhibitors. To target tumor metabolism based on metabolic characteristics may provide a new therapeutic strategy for the treatment of B cell lymphoma.
\end{abstract}

Key words: B cell lymphoma, metabolic characteristics, glycolysis, glutaminolysis, metabolic inhibitors

\section{Introduction}

Lymphoma is a group of blood cell tumors that develop from lymphocytes (a type of white blood cell). There are dozens of types of lymphomas, but the two main categories of lymphomas are Hodgkin's lymphomas (HL) and the non-Hodgkin lymphomas (NHL) [1]. About $90 \%$ of lymphomas are non-Hodgkin lymphomas [2]. A wide variety of lymphomas are in this class, and the incidence of
non-Hodgkin lymphoma increases with age. Non-Hodgkin's lymphomas (NHL) constitute a heterogeneous group of lymphoproliferative diseases, with different presenting features, clinical course and response to treatment [3]. Prognosis and treatment depends on the specific type of lymphoma as well as the stage and grade. Chemotherapy is still play an important role in treatment. However, the rapid 
evolution of drug resistance is a major obstacle for lymphoma therapy, and lead to insensitivity to chemotherapy drugs and recurrence. So, the emergence of several less toxic but highly effective agents, personalized approaches will be more important to overcome the resistance of lymphoma in the future.

Metabolic reprogramming has been proposed to be a central feature of cancer, and it is also thought to be essential for rapid cancer cell proliferation. Cancer cells exhibit profound alterations in their metabolism, such as an increase in aerobic glycolysis, first discovered in the early 20th century [4]. Characteristic metabolic changes enable cells to meet the large biosynthetic demands (more energy and macromolecules) associated with cell growth, proliferation and division. And it has been reported that cancer cells exhibit abnormal glucose and glutamine metabolism in order to promote the cell growth and survival under restrictive conditions of the tumor environment $[5,6]$. So, cancer metabolism has emerged as an important area of research in recent years. Yuan $S$ et al have been showed that glioblastoma stem cells are highly glycolytic, and a glycolytic inhibitor 3-bromo-2-oxopropionate-1propyl ester (3-BrOP) combination strategies can be used to effectively overcome their drug resistance based on their metabolic properties [7]. Bhattacharya $B$ et al demonstrated that hypoglycaemia increased resistance to cytotoxic agents, especially in tumour cells with a high dependence on glycolysis [8]. Daemen A et al used the metabolite profiling stratified 38 pancreatic ductal adenocarcinomas cell lines into 3 subtypes, and then they further demonstrated that glycolytic and lipogenic subtypes appear to use glucose and glutamine in a different manner, and they also showed distinct sensitivity to metabolic inhibitors in vitro and in vivo [9].

In this study, we hypothesize that the diverse forms of lymphoma can be stratified into different subtypes by their dependence on glucose and glutamine, and their metabolic characteristics are also distinct. And, these subtypes may also show distinct sensitivity to metabolic inhibitors. So, we focused on the 9 B-cell non-hodgkin-lymphoma (B-NHL) cell lines, including the most common subtype of diffuse large B cell lymphoma, high grade malignant mantle cell lymphoma (MCL, which is an aggressive lymphoid neoplasia derived from mature B cells and is considered incurable with current therapies), and unusual case burkitt's lymphoma. Firstly, we identified different subtypes by survival rate of these cell lines in culture under a glucose or glutamine starvation condition. And then we detected their metabolic properties, including OCR, ECAR, the proliferative capacity, glycolysis and mitochondrial function. Finally, we demonstrated B-NHL with different metabolic characteristics showed distinct sensitivity to metabolic inhibitors.

\section{Materials and Methods}

Cell Culture, Inhibitors. All cell lines (from ATCC), were cultured in RMPI 1640 (Invitrogen) containing glutamine and glucose, supplemented with 15\% (Mino), 20\% (Jeko-1) or 10\% FBS (other cell lines) (BI). For glucose/glutamine starvation experiments, cells were seeded in RMPI 1640 (Invitrogen, +glutamine, -glucose) or RMPI 1640 (Invitrogen, -glutamine) without glucose or glutamine supplemented with $10 \%$ (or $15 \%, 20 \%$ ) dialysed FBS (BI). 3-BrPA was purchased from Sigma, and BPTEs was purchased from Selleck.

Extracellular acidification rate and basal oxygen consumption rate. Oxygen consumption rates (OCR) and extracellular acidification rates (ECAR) were measured using an XF24 extracellular analyser (Seahorse Bioscience). A 24-well cell culture microplate was coated with Corning ${ }^{\circledR}$ Cell-Tak ${ }^{\mathrm{TM}}$ Cell and Tissue Adhesive (Corning Incorporated) to allow adhesion of suspended cells. Cells in the exponential growth phase were seeded in triplicate at a density of $2.5 \times 10^{5}$ cells/well in 24-well cell culture microplate. After calibration of the analyzer, sequential compound injections, including oligomycin A, carbonyl-cyanide p-trifluoromethoxyphenylhydrazone (FCCP), antimycin A and rotenone, were applied on the microplate to test mitochondrial respiration. Sequential compound injections, including glucose, oligomycin A and 2-DG, were applied to test glycolytic activity.

\section{Cell proliferation and cytotoxicity assays}

Cell survival rate and IC50 was measured using MTS assay (CellTiter 96 AQueous One Solution, Promega), and doubling time was measured by survival cell count assay (Nexcelom Cellometer $\left.{ }^{\circledR}\right)$. In brief, exponentially growing cells were seeded at $4 \times 10^{4}$ cells/well in a 96- well plate. Then cells were treated with indicated drugs, followed by continuous incubation for $72 \mathrm{~h}$. $20 \mu \mathrm{l}$ MTS was directly added to each well and the incubation was continued for an additional $3 \mathrm{~h}$. The absorbance of optical density was measured with a microplate reader (Sunrise, Tecan) at wavelength $490 \mathrm{~nm}$. Cell viability was calculated by the following formula cell viability $(\%)=$ (average absorbance of treated group-average absorbance of blank)/(average absorbance of untreated group average absorbance of blank) $\times 100 \%$. IC50 was calculated by GraphPad Prism 5 software. 


\section{Glucose consumption and lactate production}

Cells in the exponential growth phase were seeded in triplicate at a density of $5 \times 10^{5}$ cells/well in 12 -well plates. Culture media were removed after $24 \mathrm{~h}$ incubation for analysis of glucose and lactate levels by an SBA-40C Biosensor (Hayfield Ji'nan science and Technology Development Co.Ltd, China). Glucose consumption or lactate production was determined by the concentration difference between cell culture medium and fresh medium without cells, and the value was normalized to the cell number.

\section{Quantitative real-time Polymerase Chain Reaction (PCR) analysis}

The primer sequences for the genes to be measured are as follows: 5'- TTGACCAGGAGATTG ACATGGG' (forward) and 5'- CAACCGCATCAGGA CCTCA' (reverse) for HK2, 5'- AGGGTCTGTTACCT AGCTTGG-3' (forward) and 5'- ACGTTCGCAATCCT GTAGATTT -3' (reverse) for GLS. Real-time PCR analysis was performed by using the SYBR Premix Ex Taq II kit (TaKaRa Bio, Otsu, Shiga, Japan) and Real-Time PCR Detection Systems (Bio-Rad, Hercules, CA, USA).

\section{Western Blot Analysis}

Western blot analyses to detect HK2 (anti-human HK2 antibody, abcam, 1:1000) and GLS (anti-human GLS antibody, CST, 1:1000) protein levels were performed as described previously [10]. The bands were scanned using ChemiDoc ${ }^{\mathrm{TM}}$ Touch Imaging System (BD). The band intensities for HK2 and GLS were normalized to those of $\beta$-actin, and calculated using Image J software (National Institutes of Health, USA).

\section{Flow cytometry analysis of mitochondria mass}

Cells were stained with $1 \mu \mathrm{M}$ MitoTracker Red (Invitrogen) for $30 \mathrm{~min}$ at $37{ }^{\circ} \mathrm{C}$ to measure the mitochondrial mass. Analysis was performed using FACSCalibur cell sorting system (BD Biosciences, USA) and analyzed using CellQuest 3.0 software.

\section{Statistical analysis}

Where appropriate, the results are expressed as mean \pm SD. P-values of 0.05 or less were accepted as indicators of statistically significant data. Student's t-tests were conducted to compare each treatment group to every other, and adjusted by ANOVA for multiple comparisons. Each experiment was repeated 3 times.

\section{Results}

\section{Metabolic Characterization Reveals a Glucose-dependent (Glu-dependent), Glutamine-addicted (GLN-addicted) and Glutamine and Glucose equally utilized subtype (GLN=Glu).}

Cancer cells exhibit abnormal glucose and glutamine metabolism to confer growth and survival advantages under restrictive conditions of the tumor environment. We hypothesized that glutamine or glucose starvation can induce cell death. Firstly, to test this we measured the cell viability of 9 human B-NHL cell lines (survival rate) by MTS which cultured under the 3 different medium conditions (regular media, without glucose or glutamine) for $72 \mathrm{~h}$. Glutamine starvation-induced cell apoptosis significantly were named GLN-addicted subtype (Fig. 1A). Thus, we defined GLN addiction as dependency of cells on glutamine for survival. Glucose starvation-induced cell apoptosis significantly were named Gludependent subtype (Fig. 1B). The survival rate between glutamine and glucose starvation was no difference, but both significantly induced cell death named glutamine and glucose equally utilized subtype (GLN=Glu) (Fig. 1C). These results demonstrated that lymphoma cells exhibit distinct glucose and glutamine for growth and survival. Significantly, the SU-DHL-6 and SU-DHL-4 is Glu-dependent, but the dependence of SU-DHL-2 on glucose and glutamine is equally, which indicated that the dependence on glucose or glutamine is different even in the same type of lymphoma (all of these 3 cell lines are diffuse large B cell lymphoma). We all know the classification is extremely complicated for lymphoma because there are many types (about 30 types on non-Hodgkin lymphoma). Therefore, these results also indicated that the importance of classifying lymphoma to different subtypes by their metabolic characterization.

In addition, the results of figure 1 also indicated that the glucose and glutamine is very important for lymphoma cells survival and proliferation. Glucose or glutamine starvation both can induce the lymphoma cells to death significantly. But in the GLN-addicted subtype, during glucose limitation, cells still depend on a glutamine supply nutrients to proliferate and survive. All these cell lines from the GLN-addicted and Glu-dependent subtypes appear to use glucose and glutamine in a different manner and function on proliferation and survival. Which indicated that the glycolysis and the mitochondrial function between these subtypes is different. 
A
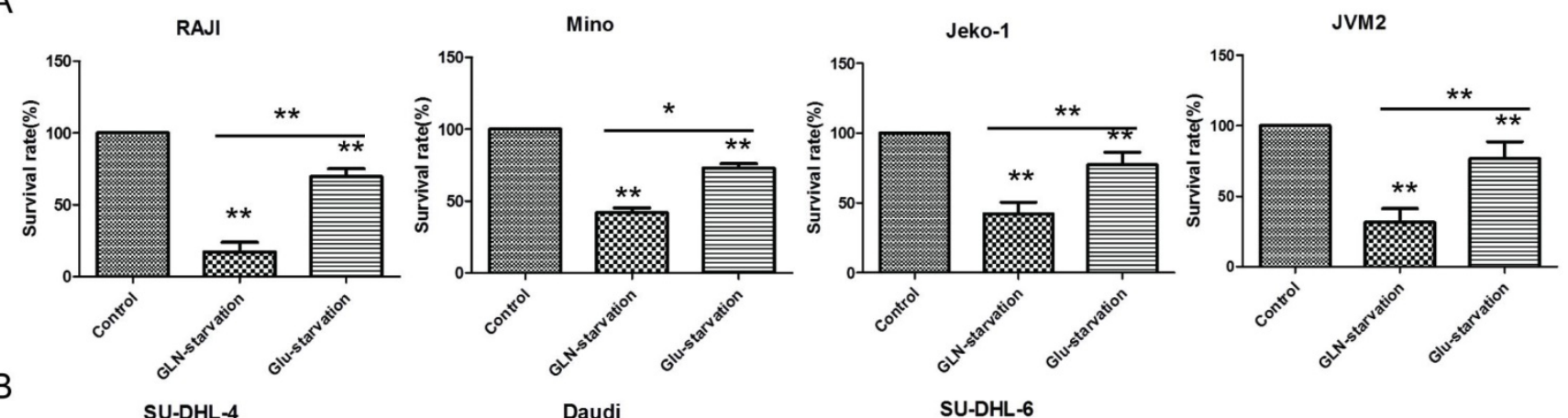

B
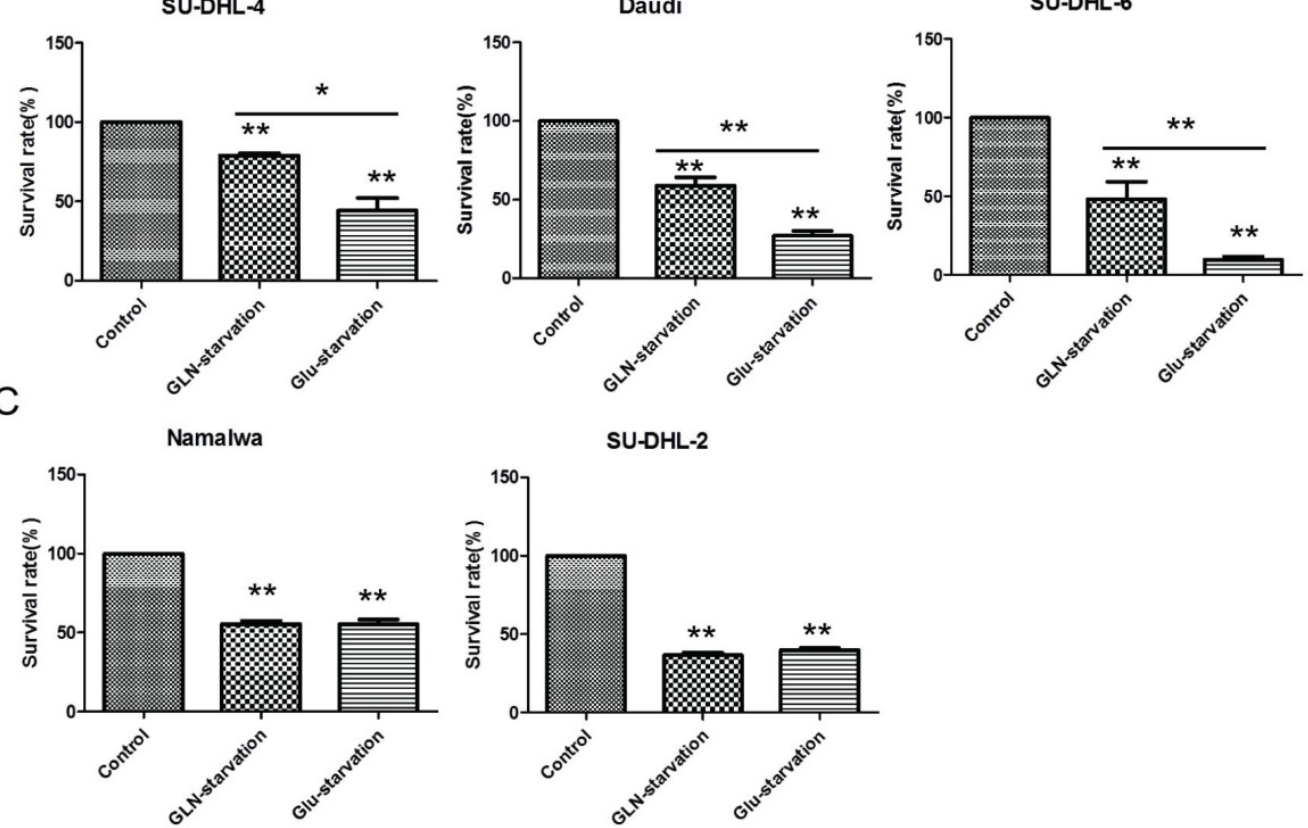

Figure 1. Identification of distinct metabolic subtypes in B cell lymphoma cell lines through their sensitivity to GLN/Glu starvation. (A) GLN addicted, (B) Glu-dependent and (C) GLN=Glu based on their sensitivity to GLN/Glu starvation. Cell death was measured after 72 hours of GLN/Glu starvation by MTS. Data are the average \pm SD of three independent cultures. $* P<0.05$, $* * P<0.001$.

\section{GLN-addicted, Glu-dependent and $\mathbf{G L N}=\mathbf{G l u}$ Subtype exhibits distinct mitochondrial function}

Oxygen consumption rates (OCR) in 9 B-NHL cell lines was examined by XF extracellular analyzer. Which can reveal the capacity of mitochondrial respiration, and it is an important parameter for mitochondrial function. The difference between these subtypes (GLN-addicted, Glu-dependent) suggested that they may differ in use of glucose and glutamine, the most abundant carbon sources available to cancer cells. We predicted that the glucose-dependence subtype would use glucose more for aerobic glycolysis, whereas the GLN-addicted subtype would use more glutamine for the tricarboxylic acid (TCA) cycle and lipid synthesis with a stronger mitochondrial function. The results consisted with our hypothesis, basal OCR value revealed a significant higher in GLN-addicted and GLN=Glu subtype compared with the Glu-dependent (Fig. 2A-B, Table 1), which indicated that GLN-addicted and GLN=Glu subtypes showed on average higher $\mathrm{O} 2$ consumption. Consistent with these observations, and a greater mitochondrial content (Mitotracker intensity) in GLN-addicted and GLN=Glu subtypes (Fig. 2D, Table 1), and mitochondrial spare respiratory capacity in these two subtypes also was high (Fig. 2C, Table 1).

Thus, cell lines from the GLN-addicted and GLN=Glu subtypes appear to use glutamine more effectively with a higher mitochondrial function. In the tumor environment, glutamine is the most important and abundant amino acid for tumors cells consumption [11, 12]. And akin to glucose, glutamine also as a major precursor for ATP production and replenishment of TCA cycle intermediates and metabolic intermediates, including synthesis of proteins, nucleic acids, lipids, hexosamines, glutathione (GSH), and NADPH [13]. So, cancer cells dependent on glutamine metabolism may benefit for growth and survival under the conditions of limited local resources in tumor environment. 


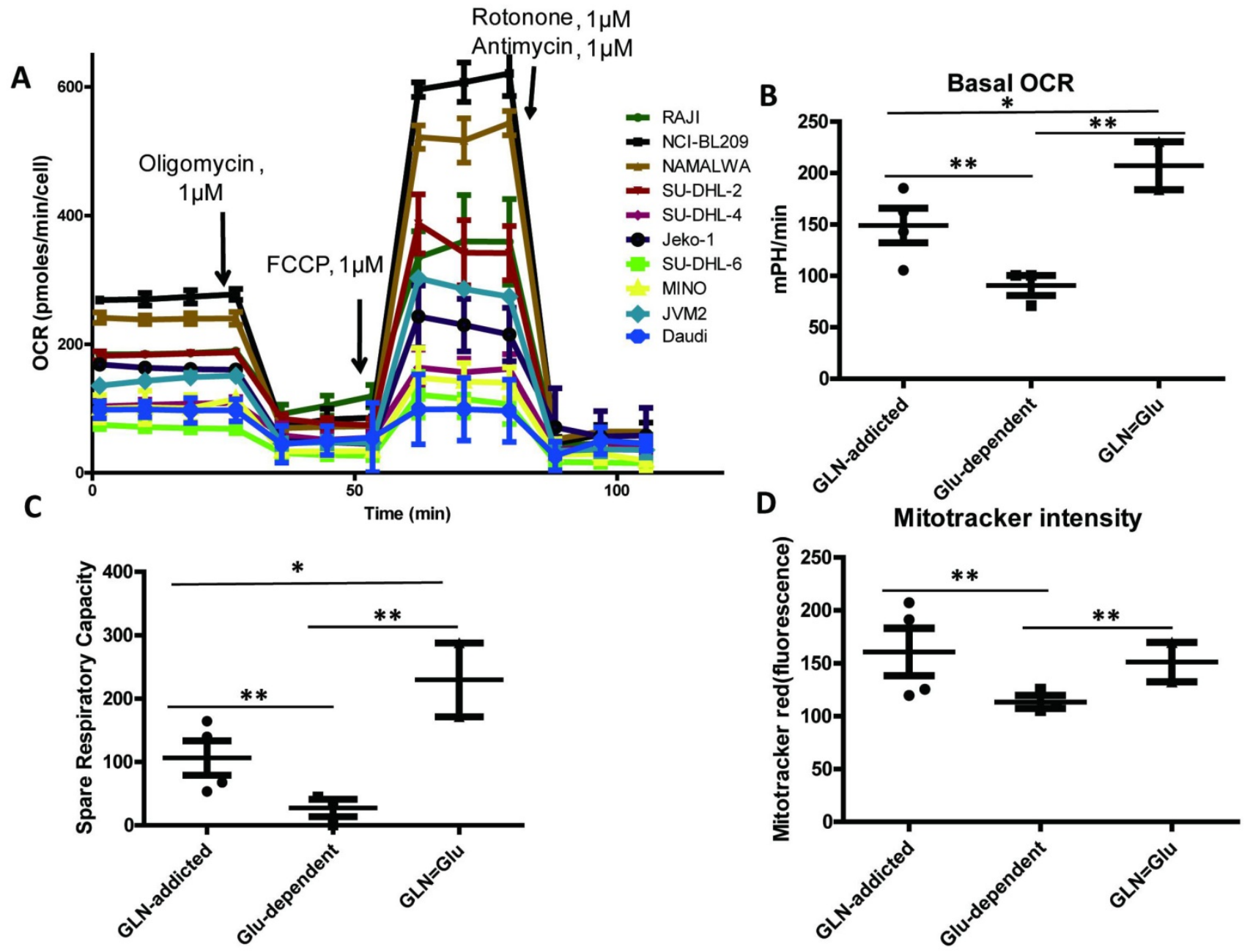

Figure 2. Mitochondrial function in B cell lymphoma cell lines. (A) Oxygen consumption rate (OCR) was monitored with a Seahorse XF24 analyzer for 100 min. The metabolic inhibitors oligomycin, FCCP, rotenone and antimycin were injected sequentially at different time points as indicated. (B) Comparison of basal oxygen consumption rates (OCRs) in 3 subtypes. (C) Spare respiratory capacity in 9 B cell lymphoma cell lines. Spare Respiratory Capacity= Maximal Respiration- Basal Respiration. (D) Comparison of relative mitochondria number (Mitotracker intensity per cell) in 3 subtypes cell lines. For B and D, the mean and SD between cell lines belonging to 3 subtypes is plotted where each cell line is shown as one dot, representing the mean of three replicates. Asterisks denote a statistically significant difference by One-Way ANOVA (*P $<0.05$, $* * P<0.001)$.

Table 1. The value of basal OCR, spare respiratory capacity and mitotracker intensity in 9 cell lines.

\begin{tabular}{llll}
\hline Cell Line & $\begin{array}{l}\text { OCR } \\
(\mathrm{pmol} / \mathrm{min})\end{array}$ & $\begin{array}{l}\text { Spare Respiratory Capacity } \\
(\mathrm{pmol} / \mathrm{min})(\text { Mean } \pm \mathrm{SD})\end{array}$ & $\begin{array}{l}\text { Mitotracker } \\
\text { intensity }\end{array}$ \\
\hline NCI-BL209 & $272.40 \pm 8.90$ & $335.60 \pm 10.03$ & $119.72 \pm 17.81$ \\
Raji & $186.12 \pm 11.67$ & $165.25 \pm 10.49$ & $125.50 \pm 11.52$ \\
Mino & $104.17 \pm 10.92$ & $30.06 \pm 9.09$ & $191.20 \pm 13.98$ \\
JVM-2 & $144.49 \pm 8.01$ & $142.91 \pm 9.79$ & $207.09 \pm 15.16$ \\
Jeko-1 & $163.32 \pm 9.45$ & $66.02 \pm 6.17$ & $207.09 \pm 15.16$ \\
SU-DHL-4 & $106.24 \pm 10.23$ & $54.35 \pm 8.20$ & $109.71 \pm 9.32$ \\
Daudi & $97.76 \pm 10.38$ & $0.365 \pm 7.52$ & $125.45 \pm 8.43$ \\
SU-DHL-6 & $70.89 \pm 10.09$ & $43.45 \pm 6.82$ & $105.26 \pm 8.31$ \\
NAMALWA & $239.78 \pm 9.05$ & $287.64 \pm 6.89$ & $132.68 \pm 10.59$ \\
SU-DHL-2 & $184.54 \pm 11.48$ & $172.37 \pm 8.66$ & $169.92 \pm 16.37$ \\
\hline & & &
\end{tabular}

\section{Different rates of glycolysis in Glu-dependent, GLN-addicted and GLN=Glu Subtype}

The extracellular acidification rates (ECAR) in 9 B-NHL cell lines was examined by XF extracellular analyzer. And the glucose consumption and lactate production was examined by the biosensing analyzer.
The ECAR value can reveal the change of anaerobic metabolism. The high glycolytic rate and increased glucose uptake of tumors were first reported by Warburg [14]. First, all the lymphoma cell lines exhibited significant up-regulation of glycolytic metabolism compared to normal B lymphoblast cell NCI-BL209 (the ECAR value is about $49.06 \pm 4.29$ $\mathrm{mpH} / \mathrm{min}$ ) after glucose injection (Fig. $3 \mathrm{~A}$ ). The cell lines in Glu-dependent and GLN=Glu subtype have enhanced glycolysis rate compared with GLN-addicted subtype, showed by higher basal ECAR value (basal ECAR: the measure of glycolysis as the ECAR rate reached by a given cell after the addition of saturating amounts of glucose) (Fig. 3B, Table 2), glucose consumption and lactate production (Fig. 3D). However, figure 3C showed that the glycolytic reserve in Glu-dependent subtype was lower than the other two (Table 2). This may result from these lines have a higher basal ECRA. So, the cell lines in the Glu-dependent subtype have a high glycolytic phenotype with a lower mitochondrial 
respiratory capacity. Generation of ATP through oxidative phosphorylation in the mitochondria is an efficient and preferred metabolic process, but when the ability of cells to generate ATP through mitochondrial oxidative phosphorylation is compromised, cells are able to increase glycolytic activity to maintain their energy supply. In addition, a hypoxic environment within the tumor is another important factor that contributes to the glycolysis [15, 16]. So inhibit the glycolysis by glucose deprivation can obviously prevent cancer cells survival and growth, especially in the Glu-dependent subtype.

A

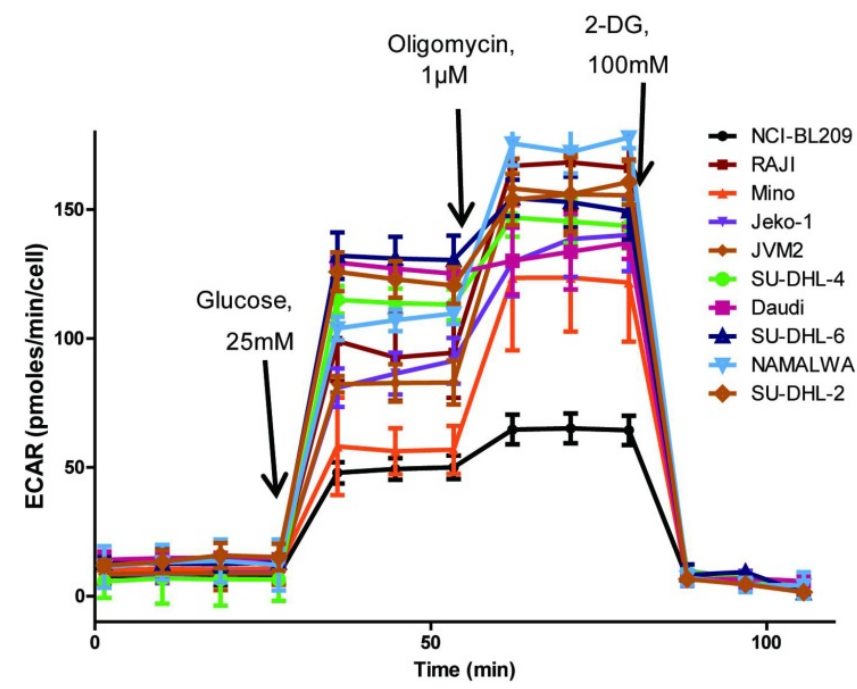

B

C
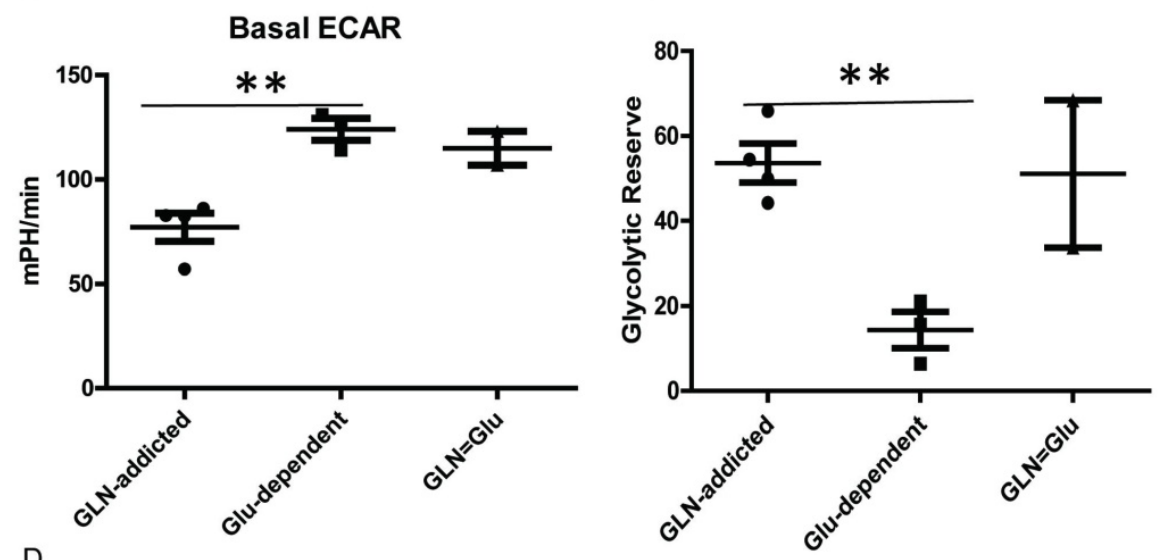

D

Glucose consumption
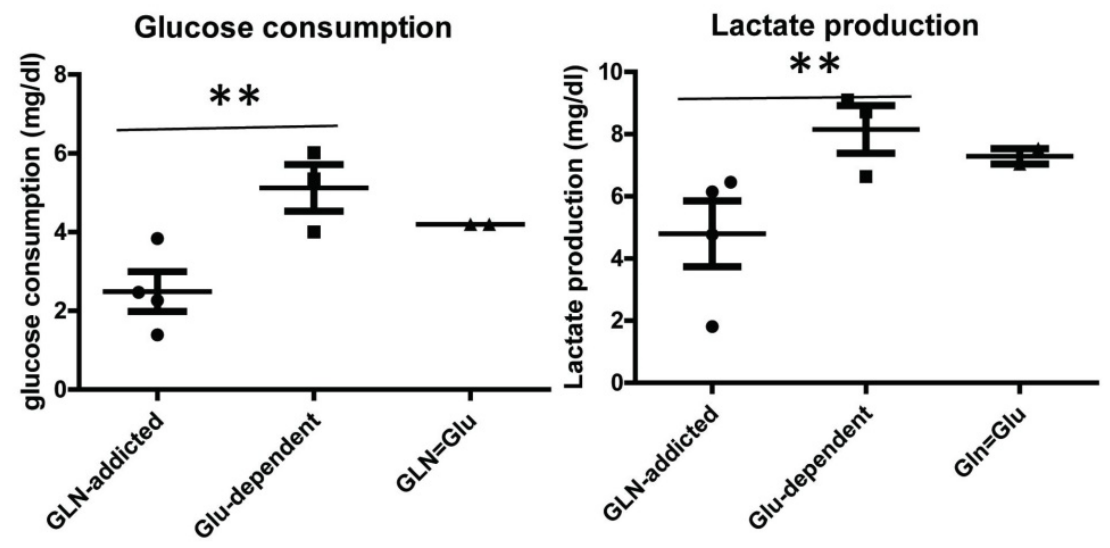

Figure 3. Glycolysis in B cell lymphoma cell lines. (A) Extra cellular acidification rate (ECAR) was monitored with a Seahorse XF24 analyzer for 100 min. The metabolic inhibitors glucose, oligomycin A and 2-DG were injected sequentially at different time points as indicated. (B) Comparison of basal ECAR in 3 subtypes. (C) Comparison of glucose consumption and lactate production in 3 subtypes cell lines. (D) Glycolytic reserve in $9 \mathrm{~B}$ cell lymphoma cell lines. Glycolytic Reserve= Glycolytic Capacity-Glycolysis. For $B$, and $\mathrm{D}$ the mean and SD between cell lines belonging to 3 subtypes is plotted where each cell line is shown as one dot, representing the mean of three replicates. Asterisks denote a statistically significant difference by One-Way ANOVA $(* \mathrm{P}<0.05, * * \mathrm{P}<0.001)$. 
Table 2. The value of basal ECAR and glycolytic reserve in 9 cell lines.

\begin{tabular}{lll}
\hline Cell Line & $\begin{array}{l}\text { ECAR }(\mathrm{pmol} / \mathrm{min}) \\
(\mathrm{Mean} \pm \mathrm{SD})\end{array}$ & $\begin{array}{l}\text { Glycolytic Reserve } \\
(\mathrm{pmol} / \mathrm{min})(\text { Mean } \pm \text { SD) }\end{array}$ \\
\hline NCI-BL209 & $49.06 \pm 4.29$ & $31.38 \pm 8.20$ \\
Raji & $92.67 \pm 16.6$ & $54.42 \pm 16.77$ \\
Mino & $57.04 \pm 12.38$ & $21.07 \pm 9.32$ \\
JVM-2 & $82.26 \pm 6.33$ & $44.26 \pm 7.29$ \\
Jeko-1 & $86.17 \pm 8.16$ & $49.94 \pm 5.84$ \\
SU-DHL-4 & $113.9 \pm 5.68$ & $15.63 \pm 5.74$ \\
Daudi & $127.19 \pm 1.51$ & $6.41 \pm 9.77$ \\
SU-DHL-6 & $131.16 \pm 8.97$ & $30.88 \pm 11.60$ \\
NAMALWA & $106.85 \pm 4.25$ & $68.44 \pm 6.99$ \\
SU-DHL-2 & $123.08 \pm 7.25$ & $33.73 \pm 11.02$ \\
\hline
\end{tabular}

\section{Differences in these three Subtypes at HK2 and GLS level}

The shift from oxidative phosphorylation (OXPHOS) to aerobe glycolysis is a hallmark of cancer cells. Cancer cells preferentially metabolize glucose into lactate for energy production, despite the presence of abundant oxygen, which is critical for cancer cell proliferation, division, migration and metastasis [17]. Hexokinases catalyse the first and irreversible step of glucose metabolism (ATPdependent phosphorylation of glucose to yield glucose-6-phosphate) [18]. Hexokinase 2 (HK2) is the only and major isozyme that is overexpressed in tumours and contributes to aerobic glycolysis, and thus it is documented as a pivotal player in the Warburg effect and is proposed as a metabolic target for cancer therapeutic development [19, 20]. So, we want to evaluate the glycolysis by detecting the HK2 mRNA and protein level in lymphoma cell lines. The result showed that HK2 (mRNA and protein) was significantly high in Glu-dependent and GLN=Glu subtype, but low in GLN-addicted subtype (Fig. 4B-D). Which indicated that the glycolytic capacity is higher in Glu-dependent and GLN=Glu subtype.

Glutaminolysis is important for metabolism and biosynthesis of cancer cells, and GLS is essential in the process. The first step of glutamine metabolism is the conversion of glutamine to glutamate and ammonia, which is catalyzed by glutaminase (GLS) [21]. GLS plays an important role in the regulation of glutamine catabolism and is a key enzyme. So we used the level of GLS to evaluate the glutaminolysis in cancer cells. Compared to the Glu-dependent subtype, the mRNA and protein level of GLS in GLN-addicted and GLN=Glu subtype was high (Fig. 4B-D), which may lead to these two subtypes more rely on glutamine. The cell lines in Glu-dependent subtype would use

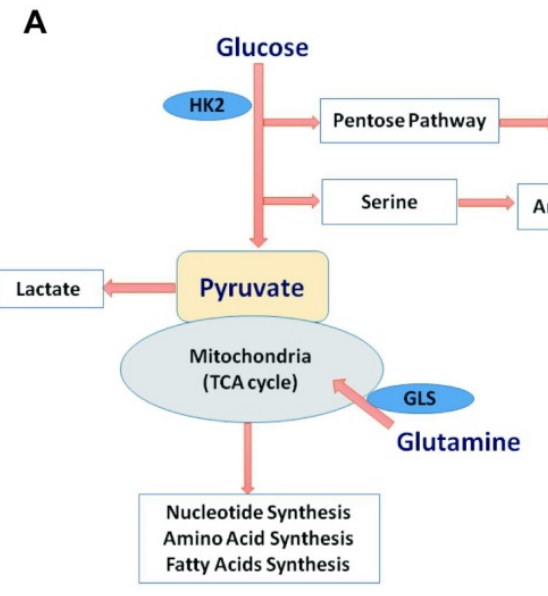

B
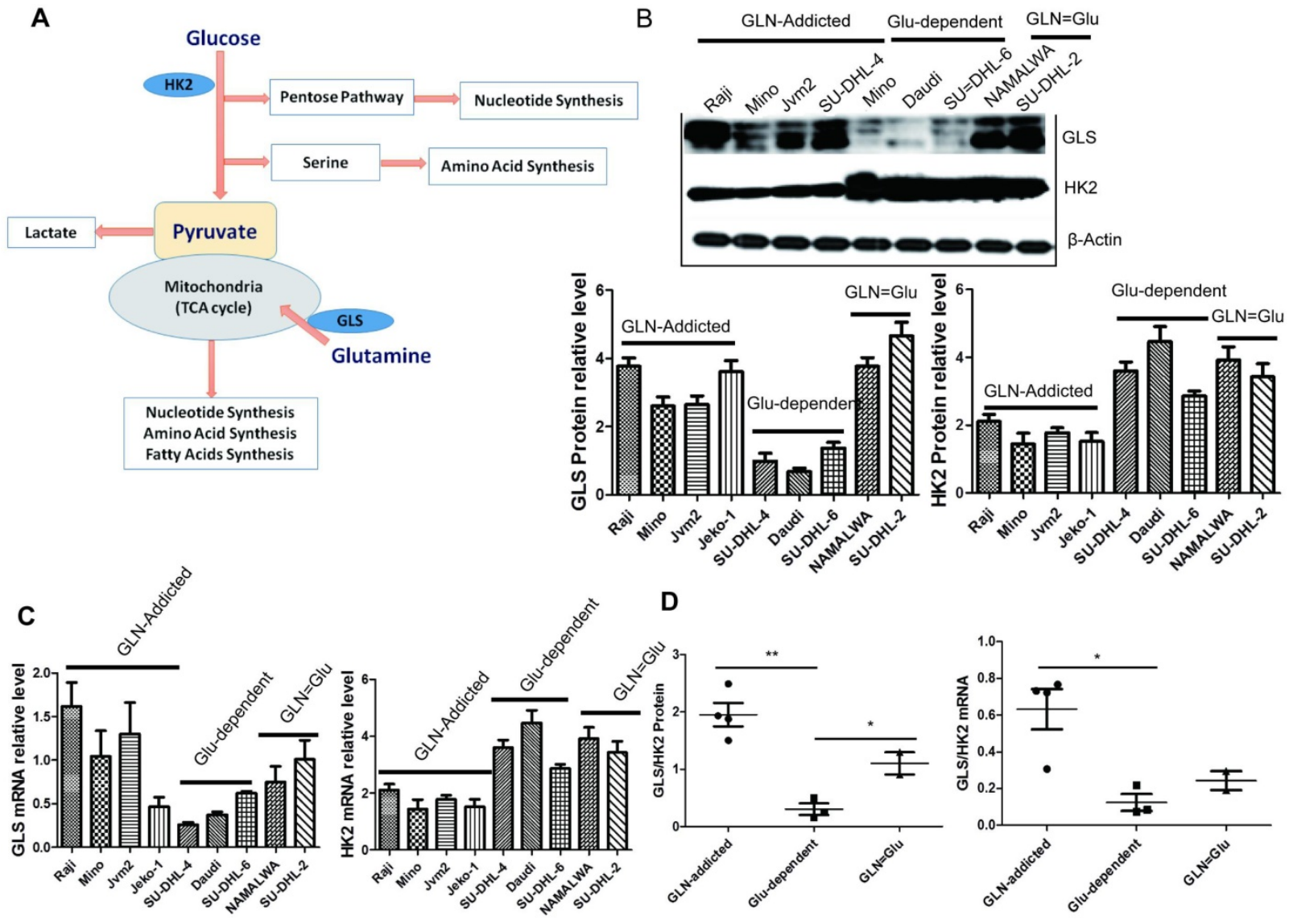

Figure 4. HK2 and GLS mRNA and protein level. (A) Model of preferential glucose and glutamine utilization in cancer cell. (B) The expression of GLS and HK2 protein level in 9 cell lines. (C) The expression of GLS and HK2 mRNA level in 9 cell lines. (D) Comparison of the ratio of GLS/HK2 protein (left) and mRNA (right) level in 3 subtypes. For $B$ the mean and SD between cell lines belonging to 3 subtypes is plotted where each cell line is shown as one dot, representing the mean of three replicates. Asterisks denote a statistically significant difference by One-Way ANOVA (*P $<0.05$, $* * \mathrm{P}<0.001$ ). 
glucose more for aerobic glycolysis to promote growth and survival with higher level of HK2 ( which can contribute to aerobic glycolysis), whereas the GLN-addicted subtype would use more glutamine for the tricarboxylic acid (TCA) cycle and lipid synthesis with a stronger glutaminase (GLS). Using the ratio of the average expression of GLS at gene or protein level to HK2 clearly distinguished NHL cell lines by metabolic dependency preference.

\section{B cell lymphoma with different metabolic characteristics show distinct sensitivities to metabolic inhibitors}

Based on their distinct metabolic wiring, alterations in energy metabolism and increased dependency on glycolysis or glutaminolysis, we predicted that Glu-dependent and GLN-addicted cell lines would show differential sensitivity to inhibitors targeting aerobic glycolysis (3-BrPA) [22] and glutaminolysis [bis-2-(5-phenylacetimido-1,2,4, thiadiazol-2-yl)ethyl sulfide (BPTES)] [23]. Indeed, as predicted, the Glu-dependent subtype was enriched for lines that were sensitive to the inhibitor 3-BrPA (Fig. 5A), whereas the GLN-addcited subtype was enriched for lines that were sensitive to inhibitors BPTES (Fig. 5B). In contrast, GLN=Glu subtype showed minimal sensitivity to 3-BrPA and BPTES (Fig. 5C). Thus, Glu-dependent and GLN-addicted subtypes are functionally distinct and show differential sensitivity to glycolytic and glutaminolytic inhibitors. The results also indicated that the utility of metabolic subtype to predict sensitivity of tumors to metabolic inhibitors. In addition, to target tumor metabolism may provide a new therapeutic strategy for the treatment of NHL or could be an efficient alternative to target metabolic characteristics in conjunction with drug resensitizes drug-resistant in NHL.

\section{Discussion}

Cancer cells possess altered metabolic systems by utilizing higher quantities of glutamine and glucose for their high proliferative and requirements. Glutamine provides cancer cells with the energy required to synthesize macromolecules [24]. And cancer cells usually obtained adenosine triphosphate (ATP) through phosphorylation to generation by means of glycolysis even in the presence of oxygen [25]. Glucose and glutamine are the most crucial substrates required for metabolism and subsequent proliferation and tumor development. Therefore, glucose is mainly utilized for glycolysis to produce ATP, glutamine is used as the mitochondrial tricarboxylic acid cycle (TCA) substrate and for nicotinamide adenine dinucleotide phosphate
(NADPH) and fatty acids synthesis [26]. Due to the largely different metabolic requirements of cancer cells, it is of great interest and importance to investigate the physiological effects of diverse forms of lymphoma on glucose and glutamine.

So, this study is the first, to our knowledge, classified $9 \mathrm{~B}$ cell lymphoma cell lines into 3 metabolic subtypes through their distinct dependence on glutamine or glucose for survival (so-called GLN-addicted, Glu-dependent and GLN=Glu) (Fig. 1) and to demonstrate that each subtype is enriched for drug sensitivity to unique classes of metabolic inhibitors (Fig. 5). But why dependence on glutamine or glucose for survival varies so much among these lymphoma cell lines, even the same lymphoma types. Here we showed that GLN-addicted and Glu-dependence cell lines were significantly different between glycolysis capacity and mitochondrial function. The cell lines in the GLN-addicted subtype are more rely on the glutamine which metabolism via TCA cycling for proliferation and survival. Glutamine metabolism contributes to biomass precursors as it is a major carbon and nitrogen source [27, 28]. One of the essential functions of mitochondria is TCA cycle. Consistent with these, GLN-addicted lines showed on average higher capacity of mitochondrial respiration (OCR), spare respiratory capacity and a greater mitochondrial content (Mitotracker intensity) (Fig. 2, Table 1). Whereas, the Glu-dependent lines have higher glycolysis rate with higher basal ECAR, glucose consumption and lactate production (Fig. 3, Table 2). Glycolytic Reserve measure indicates the capability of a cell to respond to an energetic demand as well as how close the glycolytic function is to the cell's theoretical maximum. But, unlike GLN-addicted and GLN=Glu lines, the glycolytic reserve of Glu-dependence lines is low, which may result from these cell lines with the highest basal ECAR.

It is generally thought that rates of glutamine utilization are limited by mitochondrial uptake via GLS [29]. And as HK2 is the key enzyme that determines the direction and magnitude of glucose flux, the study has been identified the glucose metabolism can be regulated by controlling HK2 activity in colon cancer cells [30]. Based on these, we used the mRNA and protein level of GLS and HK2 to evaluate the glycolysis and glutaminolysis. Results consistent with expectations, the ratio of GLS/HK2 no matter on mRNA or protein level was significantly low in Glu-dependent subtype (Fig. 4), which indicated that the glycolysis level in this group was high and more rely on glucose. On the contrary, the glutaminolysis level was high and more rely on glutamine for GLN-addicted subtype. As predicted, the Glu-dependent subtype was enriched for lines 
that were sensitive to the glycolytic inhibitor, 3-BrPA.

lines that were sensitive to glutaminolytic inhibitors, Whereas the GLN-addicted subtype was enriched for BPTEs, targeting glutaminase (Fig. 5).
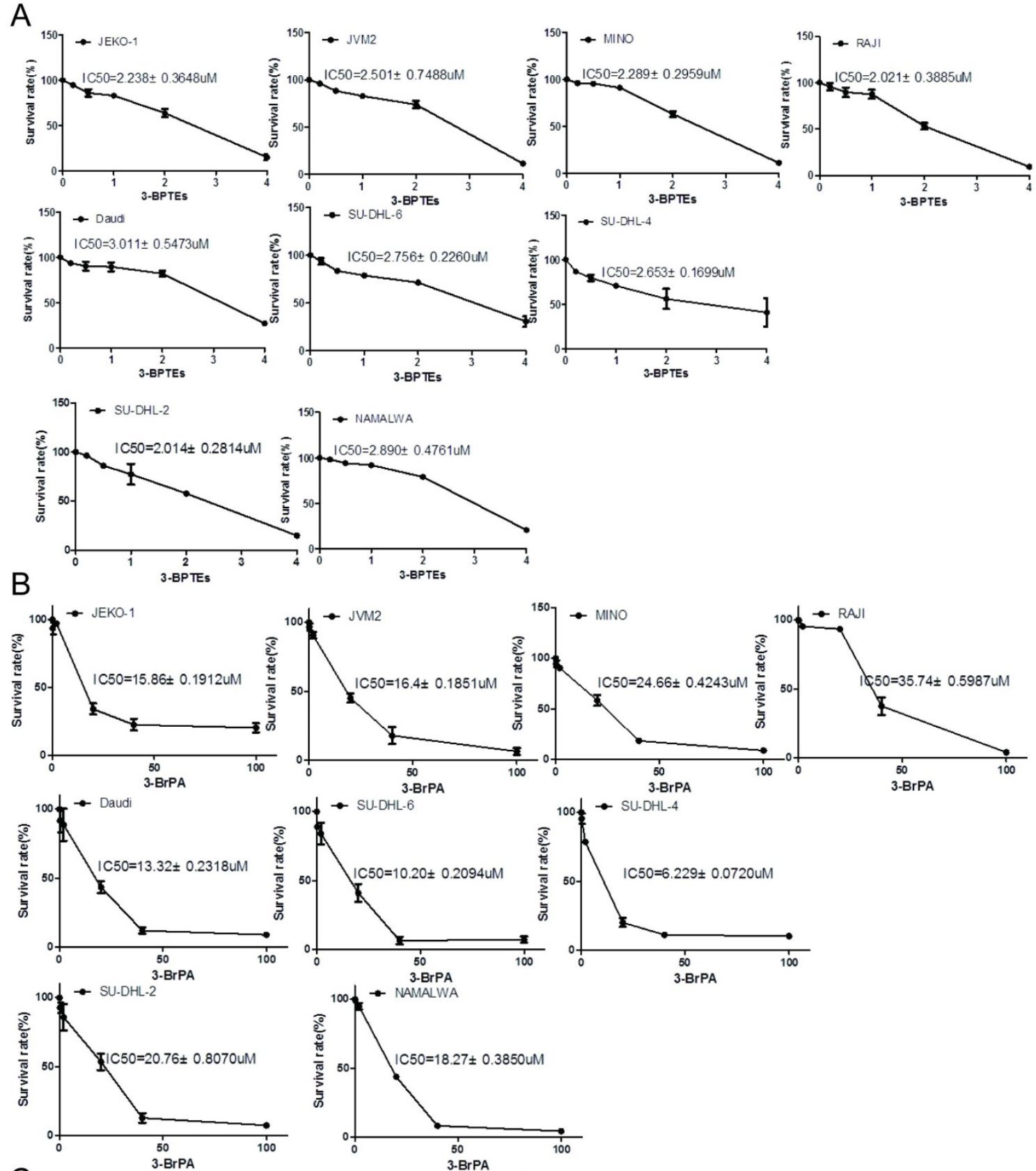

C

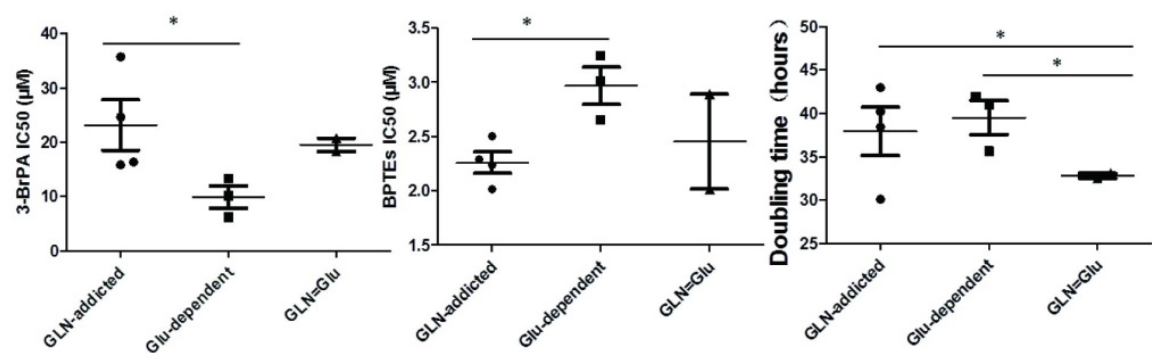

Figure 5. Response to glycolytic and glutaminolytic inhibitors in B cell lymphoma cell. (A) Cells were treated with various concentrations of glycolytic inhibitors 3-BrPA for $72 \mathrm{~h}$ and subjected to MTS assay. IC50 values in each cell line were shown above the curves. Bars, means \pm SEM, $n=3$. (B) Cells were treated with various concentrations of glutaminolytic inhibitors BPTEs for $72 \mathrm{~h}$ and subjected to MTS assay. IC50 values in each cell line were shown above the curves. Bars, means $\pm S E M, n=3$. (C) Glu-dependent associates with sensitivity to glycolytic inhibitors 3-BrPA (left) and GLN-addicted associates with sensitivity to glutaminolytic inhibitors BPTEs. (D) Doubling time for all cell lines grouped by subtype, with a higher proliferation rate for cell lines in the GLN=Glu subtype. Proliferation was measured survival cell count assay. For C and D the mean and SD between cell lines belonging to 3 subtypes is plotted where each cell line is shown as one dot, representing the mean of three replicates. Asterisks denote a statistically significant difference by One-Way ANOVA $(* \mathrm{P}<0.05$, **P $<0.001)$. 
It is worthwhile to note that the glycolysis and glutaminolysis in glutamine or glucose equally utilized subtype $(\mathrm{GLN}=\mathrm{Glu})$ both is high, reflecting by higher oxygen consumption rates (OCRs), spare respiratory capacity and mitochondria number, as well as higher glycolysis and glycolytic reserve. And this subtype was enriched for lines that were seems not sensitive to glycolytic inhibitor, 3-BrPA and glutaminolytic inhibitors, BPTEs. This may result from that these lines can obtain the energy and nutrition by glucose or glutamine metabolism for proliferation and survival. So when glycolysis was inhibited, the cells could utilize glutamine for anaplerosis, whereas glutamine starvation or blocked, the increased glucose uptake and metabolism will compensate for cell growth and survival. Cell lines in this subtype had an average doubling time that was significantly shorter. But doubling times for cell lines from the other two subtypes were more similar (Fig. 5D). Thus, this metabolic subtype has unique metabolic characteristics that are benefit for proliferation and growth. Together, these findings suggest that GLN-addicted or Glu-dependent cells depend on glutamine or glucose to promote their growth and survival, highlighting the potential for combined treatment using glutamine or glucose depletion and glutaminolytic or glycolytic inhibitors as an efficient strategy for the treatment, respectively. However, since GLN=Glu cells appear to be impervious to this treatment, the successful execution of this approach will likely depend on identification of GLN-addicted or Glu-dependence. Although our findings are based on in vitro, just cell models, we believe that they provide important insights into tumor cell metabolism in the context of Glu-dependent versus GLN-addicted.

\section{Acknowledgements}

The study was supported by National Natural Science Foundation of China (NO. 81602609), China Postdoctoral Science Foundation (2016M602593) and Science and Technology Planning Project of Guangdong Province (2017ZC0109).

\section{Competing Interests}

The authors have declared that no competing interest exists.

\section{References}

1. Bardia A, Seifter EJ. Johns Hopkins Patients' Guide to Lymphoma. Burlington, USA: Jones and Bartlett Publishers; 2010.

2. World Cancer Report 2014. World Health Organization. 2014. pp. Chapter 5.13 .

3. Mazur G, Butrym A, Kryczek I, et al. Decreased expression of CXCR4 chemokine receptor in bone marrow after chemotherapy in patients with non-Hodgkin lymphomas is a good prognostic factor. PLoS One. 2014; 9:e98194.
4. Warburg O, Wind F, Negelein E. The metabolism of tumors in the body. J Gen Physiol. 1927; 8:519-530.

5. Cantor JR, Sabatini DM. Cancer cell metabolism: one hallmark, many faces. Cancer discovery. 2012; 2:881-898.

6. Boroughs LK, DeBerardinis RJ. Metabolic pathways promoting cancer cell survival and growth. Nature cell biology. 2015; 17:351-359.

7. Yuan S, Wang F, Chen G, et al. Effective Elimination of Cancer Stem Cells by a Novel Drug Combination Strategy. Stem Cells. 2013; 31:23-34.

8. Bhattacharya B, Low SH, Soh C, et al. Increased drug resistance is associated with reduced glucose levels and an enhanced glycolysisphenotype. Br J Pharmacol. 2014; 171:3255-67.

9. Daemen A, Peterson D, Sahu N, et al. Metabolite profiling stratifies pancreatic ductal adenocarcinomas into subtypes with distinct sensitivities to metabolic inhibitors. Proc Natl Acad Sci U S A. 2015; 112:E4410-7.

10. Huang A, Ju HQ, Liu K, et al. Metabolic alterations and drug sensitivity of tyrosine kinase inhibitor resistant leukemia cells with a FLT3/ITD mutation. Cancer Lett. 2016; 377:149-57.

11. Herranz D, Ambesi-Impiombato A, Sudderth J, et al. Metabolic reprogramming induces resistance to anti-NOTCH1 therapies in T cell acute lymphoblastic leukemia. Nat Med. 2015; 21: 1182-1189.

12. Yuneva MO, Fan TW, Allen TD, et al. The metabolic profile of tumors depends on both the responsible genetic lesion and tissue type. Cell Metab. 2012; 15:157-170.

13. Gao P, Tchernyshyov I, Chang TC, et al. c-Myc suppression of miR-23a/b enhances mitochondrial glutaminase expression and glutamine metabolism. Nature. 2009; 458:762-765.

14. Warburg O. On respiratory impairment in cancer cells. Science. 1956; 124: 269-270.

15. Guppy M. The hypoxic core: a possible answer to the cancer paradox. Biochem Biophys Res Commun. 2002; 299:676-80.

16. Xu RH, Pelicano H, Zhou Y, et al. Inhibition of Glycolysis in Cancer Cells: A Novel Strategy to Overcome Drug Resistance Associated with Mitochondrial Respiratory Defect and Hypoxia. Cancer Res. 2005; 65:613-21.

17. Yang J, Li J, Le $Y$, et al. PFKL/miR-128 axis regulates glycolysis by inhibiting AKT phosphorylation and predicts poor survival in lung cancer. Am J Cancer Res. 2016; 6:473-85.

18. Robey RB, Hay N. Mitochondrial hexokinases, novel mediators of the antiapoptotic effects of growth factors and Akt. Oncogene. 2006; 25: 4683-4696.

19. Mathupala SP, Ko YH, Pedersen PL. Hexokinase-2 bound to mitochondria: cancer's stygian link to the "Warburg Effect" and a pivotal target for effective therapy. Semin Cancer Biol. 2009; 19: 17-24.

20. Vander Heiden MG. Targeting cancer metabolism: a therapeutic window opens. Nat Rev Drug Discov. 2011; 10: 671-684

21. Zhao J, Zhou R, Hui K, et al. Selenite inhibits glutamine metabolism and induces apoptosis by regulating GLS1 protein degradation via APC/C-CDH1 pathway in colorectal cancer cells. Oncotarget. 2016. [Epub ahead of print]

22. Konstantakou EG, Voutsinas GE, Velentzas AD, et al. 3-BrPA eliminates human bladder cancer cells with highly oncogenic signatures via engagement of specific death programs and perturbation of multiple signaling and metabolic determinants. Mol Cancer. 2015; 14:135.

23. Elgogary $\mathrm{A}, \mathrm{Xu} \mathrm{Q}$, Poore $\mathrm{B}$, et al. Combination therapy with BPTES nanoparticles and metformin targets the metabolic heterogeneity of pancreatic cancer. Proc Natl Acad Sci USA. 2016; 113:E5328-36.

24. Chen L, Cui H, Fang J, et al. Glutamine deprivation plus BPTES alters etoposide- and cisplatin-induced apoptosis in triple negative breast cancer cells. Oncotarget. 2016; 7:54691-54701.

25. Cairns RA, Harris IS, Mak TW. Regulation of cancer cell metabolism. Nat Rev Cancer. 2011; 11:85-95.

26. Lozy F, Karantza VG. Autophagy and cancer cell metabolism. Semin Cell Dev Biol. 2012; 23:395-401.

27. Le A, Lane AN, Hamaker $\mathrm{M}$, et al. Glucose-independent glutamine metabolism via TCA cycling for proliferation and survival in Bcells. Cell Metab. 2012; 15:110-21.

28. Lunt SY, Vander Heiden MG. Aerobic glycolysis: meeting the metabolic requirements of cell proliferation. Annu Rev Cell Dev Biol. 2011; 27:441-64.

29. Csibi A, Lee G, Yoon SO, et al. The mTORC1/S6K1 pathway regulates glutamine metabolism through the eIF4B-dependent control of c-Myc translation. Curr Biol. 2014; 24:2274-80.

30. Xiong X, Wen YA, Mitov MI, et al. PHLPP regulates hexokinase 2-dependent glucose metabolism in colon cancer cells. Cell Death Discov. 2017; 3:16103. 